\title{
Endurance exercise and the production of growth hormone and haematopoietic factors in patients with anaemia
}

\section{F Dimeo, W Knauf, D Geilhaupt, D Böning}

Br J Sports Med 2004;38:e37 (http://www.bjsportmed.com/cgi/content/full/38/6/e37). doi: 10.1136/bjsm.2004.011908

\begin{abstract}
Background: Physical activity has been shown to stimulate haematopoiesis in patients with anaemia due to chronic renal failure or haematological malignancies.

Objective: To evaluate the effect of moderate exercise on the production of haematopoietically active factors.

Methods: Ten patients (four men and six women, mean (SD) age 51 (10) years) with a haemoglobin concentration under $130 \mathrm{~g} / \mathrm{l}$ (men) or $120 \mathrm{~g} / \mathrm{l}$ (women) carried out five three minute exercise bouts at an intensity of $80 \%$ of the maximal heart rate, corresponding to a lactate concentration of 3 (0.5) $\mathrm{mmol} / \mathrm{l}$. Patients rested for three minutes between bouts. The concentrations of interleukin 6, stem cell factor, granulocyte-monocyte colony stimulating factor, granulocyte colony stimulating factor, erythropoietin, and growth hormone $(\mathrm{GH})$ were evaluated before and in the eight hours after exercise.

Results: GH had risen significantly 15 minutes after exercise (1.1 (1.3) v $2.7(2.8) \mathrm{ng} / \mathrm{ml} ; \mathrm{p}<0.05)$. No change in the concentration of the other cytokines and growth factors was observed in the eight hours after exercise.

Conclusions: In patients with anaemia, submaximal exercise does not affect the concentration of haematopoietically active cytokines. However, it leads to an increased concentration of $\mathrm{GH}$. This may be responsible for the improved haematopoiesis observed after an exercise programme in patients with chronic diseases.
\end{abstract}

ntense or prolonged exercise has been shown to affect the concentration of several cytokines and hormones that regulate the self renewal, proliferation, and maturation of blood stem cells. An increased concentration of tumour necrosis factor $\alpha$, interleukin $1 \beta$, interleukin 6 , interleukin 1 receptor antagonist, and granulocyte colony stimulating factor (G-CSF) has been observed after long lasting, strenuous exertion. ${ }^{1-4}$ Exercise induced changes in the activity of these factors may influence bone marrow function. However, studies in patients with anaemia due to chronic diseases (cancer and renal failure) suggest that exercise at moderate intensity may also affect haematopoiesis. ${ }^{5-8}$ The mechanism underlying the improvement of haematopoiesis in these patients is not known. In these studies, training was carried out at an intensity below $75 \%$ of the maximal oxygen uptake. To our knowledge, no study has evaluated the effect of moderate workloads on the production, release, and reuptake of haematopoietically active factors in patients.

\section{PATIENTS AND METHODS}

Ten patients admitted to hospital for the treatment of a haematological malignancy participated in the study (table 1). Inclusion criteria were age 18-50, normal renal and hepatic function, stable clinical status, thrombocyte concentration above 20/nl, and normocytic-normochromic anaemia with haemoglobin concentration below $130 \mathrm{~g} / \mathrm{l}$ in men and $120 \mathrm{~g} / \mathrm{l}$ in women. Exclusion criteria were fever or other signs of infection in the two days before and after the workout, pain, concurrent disease, immunosupression, treatment with corticoids, and chemotherapy or transfusion of blood products in the week preceding the workout. The study was approved by the institutional ethics committee, and all patients provided informed consent.

Patients were evaluated with a stress test on the treadmill using a Bruce protocol until exhaustion with continuous electrocardiographic monitoring and assessment of lactate concentrations and maximal oxygen uptake. Two days after this test, a workout was carried out. After a low speed three minute warm up, patients walked for three minutes on a treadmill at an intensity corresponding to $80 \%$ of the maximal heart rate. Lactate concentrations were assessed in capillary blood at the end of the workload. After this exercise bout, patients walked for three minutes at a low speed to recover. The procedure was repeated five times. Thus patients walked at an intensity of $80 \%$ of the maximum heart rate for a total of 15 minutes; the duration of training was 27 minutes. The subjective intensity of exertion was assessed using the Borg rate of perceived exertion scale.. On this scale, the intensity of exertion corresponded to 13 (somewhat hard). After training, the patients walked at a reduced speed for three minutes to cool down.

Blood samples were obtained from a central venous access before exercise and after 15, 30, 45, 60, 120, 240, and 480 minutes. Samples were immediately centrifuged and plasma was frozen at $-20^{\circ} \mathrm{C}$ until processing.

The concentration of the haematopoietically active cytokines erythropoietin (detection limit $0.6 \mathrm{pg} / \mathrm{ml}$ ), granulocytemonocyte colony stimulating factor (GM-CSF, detection limit $0.8 \mathrm{pg} / \mathrm{ml}$ ), G-CSF (detection limit $20 \mathrm{pg} / \mathrm{ml}$ ), stem cell factor (c-kit ligand, detection limit $9.0 \mathrm{pg} / \mathrm{ml}$ ), and interleukin 6 (detection limit $2 \mathrm{pg} / \mathrm{ml}$ ) were assessed using a solid phase sandwich enzyme linked immunosorbent assay (ELISA) according to the manufacturer's instructions. Human growth hormone $(\mathrm{GH}$, detection limit $0.02 \mathrm{ng} / \mathrm{ml})$ was quantified using a chemiluminescent immunometric assay (Nichols Institute Diagnostic, San Clemente, California, USA). Intraassay and interassay coefficients of variation for all enzyme immunoassays were less than $5 \%$ and $10 \%$ respectively.

The concentrations of cytokines and growth factors before and after exercise were compared using analysis of variance for repeated measures. Differences between time points were compared post hoc with the Dunnet's multiple comparison test. A value of $\mathrm{p}<0.05$ was considered significant.

Abbreviations: G-CSF, granulocyte colony stimulating factor; GH, growth hormone; GM-CSF, granulocyte-monocyte colony stimulating factor 


\begin{tabular}{|c|c|}
\hline Age (years) & $51(10)$ \\
\hline $\operatorname{Sex}(M / F)$ & $4 / 6$ \\
\hline \multicolumn{2}{|l|}{ Disease } \\
\hline Aplastic anaemia & 2 \\
\hline Non-Hodgkin lymphoma & 3 \\
\hline Acute lymphatic leukaemia & 1 \\
\hline Multiple myeloma & 2 \\
\hline Acute myeloid leukaemia & 2 \\
\hline Haemoglobin concentration (g/l) & $99(18)$ \\
\hline White blood cell count (cells/nl) & $3.1(2.2)$ \\
\hline Thrombocyte count (cells/nl) & $133(89)$ \\
\hline
\end{tabular}

\section{RESULTS}

The mean (SD) walking speed during the intensive workloads was $4.5(0.5) \mathrm{km} / \mathrm{h}$ (range 3.5-5.5) and the mean walking distance $1.32(0.4) \mathrm{km}$ (range 780-2000 m). The mean heart rate was 135 (9) beats/min, corresponding to 77 (5)\% of the maximum cardiac frequency; the mean lactate concentration was $2.4(0.5) \mathrm{mmol} / \mathrm{l}$. The intensity of effort on the Borg scale corresponded to a score of 13 (1).

As expected, baseline concentrations of erythropoietin showed a substantial correlation with the severity of anaemia (calculated as the difference between expected and actual haemoglobin concentration, $r=0.50$ ). Concentrations of erythropoietin (basal concentration 100 (49) pg/ml), GMCSF (65 (63) pg/ml), G-CSF (65 (58) pg/ml), and stem cell factor (902 (55) pg/ml) showed no significant changes in the eight hours after physical exertion ( $p>0.05$ for all values; table 2). However, the concentration of $\mathrm{GH}$ had increased 1.5-fold 15 minutes after exercise ( 1.1 (1.3) v 2.7 (2.8) ng/ml; $\mathrm{p}<0.05)$ and returned to the initial values after 60 minutes (table 2, fig 1). Seven patients had interleukin 6 concentrations below the detection limit $(2 \mathrm{pg} / \mathrm{ml})$ in all evaluations. Mean packed cell volume and haemoglobin and creatinine concentrations remained unchanged in the eight hours after the training session, suggesting no relevant change in plasma volume after exercise.

\section{DISCUSSION}

Haematopoiesis is a complex process, which is affected by the interaction of several hormones, cytokines, and growth factors. In this study we detected a significant increase in the concentration of $\mathrm{GH}$ after exercise in anaemic patients. Several in vivo and in vitro studies have shown that GH directly or indirectly (through the secondary mediator insulin-like growth factor) stimulates the myeloid and erythroid progenitor colonies and has a regulatory effect on haematopoiesis. ${ }^{10} 11$ Exhausting or prolonged exercise is known to increase the release of GH. However, recent studies suggest that there is a linear correlation between the intensity of exertion and the production of GH. ${ }^{12}$ Our results support this hypothesis and show that, in patients with chronic

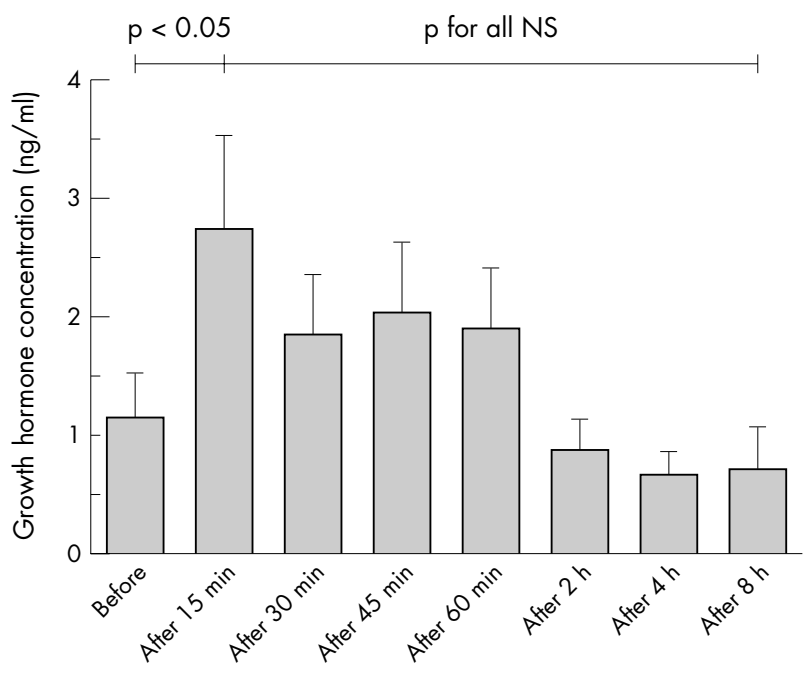

Figure 1 Concentration of growth hormone before and after exercise. Values are expressed as mean (SE) $(n=10)$.

disease, even moderate intensity exertion may substantially increase the production of GH.

Studies evaluating the changes in the concentration of erythropoietin after physical activity have yielded contradictory results. ${ }^{313}$ Our findings do not support a substantial role for erythropoietin in the exercise related activation of haematopoiesis observed in patients with chronic disease. Furthermore, studies on the effects of exercise in patients with chronic renal failure suggest that other factors are involved, as the damage to kidney parenchyma results in impairment of erythropoietin production.

Some cytokines with haematopoietic activity are produced by lymphocytes after stimulation by primed macrophages. In our study, four patients had leucopenia (absolute white blood cell count of less than $1500 / \mathrm{ml}$ ) caused by treatment or disease. Thus we did not evaluate the effect of exercise on interleukin 3, a cytokine with pleiotropic functions on haematopoiesis which is produced by $\mathrm{T}$ lymphocytes. Not much is known about the production of other haematological growth factors (G-CSF, GM-CSF, and macrophage colony stimulating factor) during leucopenia. These cytokines are produced by $\mathrm{T}$ cells but also by fibroblasts and endothelial cells. We cannot rule out the possibility that cytokines produced by $\mathrm{T}$ lymphocytes and monocytes play a role in the exercise related stimulation of haematopoiesis. However, we have previously reported that endurance exercise reduces the duration of aplasia in patients after high dose chemotherapy. ${ }^{7}$ As all patients who participated in that study had severe leucopenia (white blood cell count less than 1000/ml), an effect of the lymphocyte produced cytokines on the observed outcomes is unlikely.

Table 2 Changes in cytokine concentrations in the eight hours after exercise

\begin{tabular}{|c|c|c|c|c|c|c|c|c|}
\hline & Before & $15 \mathrm{~min}$ & $30 \mathrm{~min}$ & $45 \mathrm{~min}$ & $60 \mathrm{~min}$ & 2 hours & 4 hours & 8 hours \\
\hline $\mathrm{GH}(\mathrm{ng} / \mathrm{ml})$ & $1.1(1.7)$ & $2.7(2.8)$ & $1.8(1.8)$ & $2.0(2.1)$ & $1.9(1.8)$ & $0.8(0.9)$ & $0.6(0.6)$ & $0.18(1.3)$ \\
\hline EPO (pg/ml) & $100(49)$ & 101 (49) & $97(47)$ & $98(46)$ & $96(45)$ & $97(46)$ & $96(47)$ & $103(42)$ \\
\hline G-CSF (pg/ml) & $65(58)$ & $64(58)$ & $68(59)$ & 67 (57) & $68(58)$ & $67(60)$ & 62 (59) & $67(61)$ \\
\hline GM-CSF (pg/ml) & $65(63)$ & $65(62)$ & $60(59)$ & $57(58)$ & $57(55)$ & $58(55)$ & $58(54)$ & $60(54)$ \\
\hline $\mathrm{SCF}(\mathrm{pg} / \mathrm{ml})$ & $902(255)$ & 919 (271) & $885(263)$ & $885(251)$ & $886(239)$ & $903(215)$ & 882 (219) & $883(248)$ \\
\hline
\end{tabular}

Values are expressed as mean (SD) ( $\mathrm{n}=10$ ).

$\mathrm{GH}$, Growth hormone; EPO, erythropoietin; GM-CSF, granulocyte-monocyte colony stimulating factor; G-CSF, granulocyte colony stimulating factor; SCF, stem cell factor. 
The mechanism of exercise on haematopoiesis may also not be related to increased production or release of stimulating cytokines. In patients with chronic disease, anaemia can be a result of the long lasting inflammatory reaction. During inflammation, increased concentrations of tumour necrosis factor, interleukin 1, and other pro-inflammatory cytokines cause a defect in iron transport and metabolism thereby impairing the synthesis of haemoglobin. It has been shown that exercise may reduce the concentration of proinflammatory cytokines in patients with chronic heart failure. ${ }^{14}$ It remains to be seen whether this mechanism also reduces anaemia in patients with other chronic diseases.

We conclude the following. (a) The effects of an exercise programme on haematopoiesis in patients with chronic disease are probably not related to increased production of haematopoietically active cytokines and growth factors. $(b)$ In patients with anaemia, submaximal short duration exercise results in a significant increase in GH, which may partially explain the effects of physical activity on haematopoiesis in patients with chronic disease.

\section{Authors' affiliations \\ D Geilhaupt, D Böning, Department of Sports Medicine, Charité Universitätsmedizin Berlin, Campus Benjamin Franklin, Hindenburgdamm 30, 12200 Berlin, Germany F Dimeo, W Knauf, Department of Haematology, Oncology and Transfusion Medicine, Charité Universitätsmedizin Berlin}

Correspondence to: Dr Dimeo, Department of Haematology, Oncology and Transfusion Medicine, Charité-Universitätsmedizin Berlin, Campus Benjamin Franklin, Hindenburgdamm 30, 12200 Berlin, Germany; fernando.dimeo@charite.de

Accepted 29 March 2004

\section{REFERENCES}

1 Pedersen BK, Ostrowski K, Rohde T, Bruunsgaard H. The cytokine response to strenuous exercise. Can J Physiol Pharmacol 1998;76:505-11.

2 Pedersen BK, Steensberg A, Schierling P. Exercise and interleukin-6. Curr Opin Hematol 2001;8:137-41.

3 Schobersberger W, Hobisch-Hagen P, Fries D, et al. Increase in immune activation, vascular endothelial growth factor and erythropoietin after an ultramarathon run at moderate altitude. Immunobiology 2000;201:611-20.

4 Smith LL, Anwar A, Fragen M, et al. Cytokines and cell adhesion molecules associated with high-intensity eccentric exercise. Eur J Appl Physiol 2000;82:61-7.

5 Goldberg AP, Geltman EM, Gavin JR III, et al. Exercise training reduces coronary risk and effectively rehabilitates hemodialysis patients. Nephron 1986;42:311-16.

6 Hagberg JM, Goldberg AP, Ehsani AA, et al. Exercise training improves hypertension in hemodialysis patients. Am J Nephro 1983;3:209-12.

7 Dimeo F, Fetscher S, Lange W, et al. Effects of aerobic exercise on the physical performance and incidence of treatment-related complications after high-dose chemotherapy. Blood 1997;90:3390-4.

8 Dimeo F, Tilmann $\mathrm{MH}$, Bertz $\mathrm{H}$, et al. Aerobic exercise in the rehabilitation of cancer patients after high dose chemotherapy and autologous peripheral stem cell transplantation. Cancer 1997;79:1717-22.

9 Borg G. Perceived exertion as an indicator of somatic stress. Scand J Rehabil Med 1970;3:92-8.

10 Tian ZG, Woody MA, Sun R, et al. Recombinant human growth hormone promotes hematopoietic reconstitution after syngeneic bone marrow transplantation in mice 3. Stem Cells 1998;16:193-9.

11 Murphy WJ, Tsarfaty G, Longo DL. Growth hormone exerts hematopoietic growth-promoting effects in vivo and partially counteracts the myelosuppressive effects of azidothymidine. Blood 1992;80:1443-7.

12 Wideman L, Weltman JY, Hartman ML, et al. Growth hormone release during acute and chronic aerobic and resistance exercise: recent findings. Sports Med 2002;32:987-1004.

13 Schmidt W, Eckardt KU, Hilgendorf A, et al. Effects of maximal and submaximal exercise under normoxic and hypoxic conditions on serum erythropoietin level. Int J Sports Med 1991;12:457-61.

14 Adamopoulos S, Parissis J, Kroupis C, et al. Physical training reduces peripheral markers of inflammation in patients with chronic heart failure. Eur Heart J 2001;22:791-7. 